\title{
5. Regionaler Forschungskongress der Psychiatrischen und Neurologischen Fachkrankenhäuser Bayerns
}

Autoren

Institut
Helmfried E. Klein, Bernd Ibach

Klinik und Poliklinik für Psychiatrie, Psychosomatik und Psychotherapie, Bezirksklinikum Regensburg
Bibliografie

DOI 10.1055/s-2006-951958

Psychiat Prax 2007; 34,

Supplement 1

(c) Georg Thieme Verlag KG

Stuttgart · New York .

ISSN 1611-8332

Korrespondenzadresse Dr. med. Bernd lbach Klinik für Psychiatrie, Psychosomatik und Psychotherapie, Bezirksklinikum Regensburg Universitätsstraße 84 93053 Regensburg bernd.ibach@medbo.de

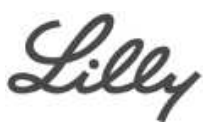

JANSSEN-CILAG

[Zukunftsarbeit]

Bristol-Myers Squibb

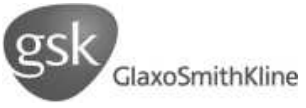

Sehr geehrter Leser,

Sie halten gerade das "Schwergewicht“ des 4. Sonderbandes vom 5 . Fachkongresses der bayerischen Kliniken für Psychiatrie und Neurologie in Ihren Händen. 71 Wissenschaftler aus bayerischen Bezirkskrankenhäusern und den assoziierten Universitätskliniken aus Günzburg und Regensburg stellen Ihnen in Form von kurzen wissenschaftlichen Vollpublikationen ihre aktuellen Forschungsaktivitäten strukturiert vor. Präsentiert und diskutiert wurden diese Ergebnisse auf dem vergangenen Irsee-Kongress in Form von Posterbeiträgen und Vorträgen. Diese Myriade von Daten, die überwiegend aus psychiatrischen Versorgungskrankenhäusern stammt, mag Sie überraschen, ist aber bei genauerem Hinsehen durchaus plausibel. Die beteiligten Kliniken stehen nämlich für etwa 10000 psychiatrische Betten bzw. für etwa 150000 behandelte Fälle pro Jahr. Diese Patienten werden von hochmotivierten Ärzten und Psychologen versorgt, die ihre Aufgabe nicht nur in der Patientenversorgung, sondern auch in der wissenschaftlichen Arbeit sehen. Das ist in Anbetracht der ansteigenden Arbeitsbelastungen und abnehmenden Personalzahlen in den Kliniken eine extreme Herausforderung. Dazu bedarf es verantwortungsbewusster Individualisten. Verantwortungsbewusstsein deshalb, weil sie damit ihre Erfahrung aus der klinischen Versorgung Anderen zugänglich machen. Individualismus, weil sie mit ihrer Arbeitshaltung ihren ganz persönlichen Weg gehen, um trotz den sehr restriktiven Rahmenbedingungen wissenschaftliches Interesse mit den hohen Anforderungen in der Patientenversorgung in Einklang zu bringen. Dieses wissenschaftliche Engagement trägt erheblich zu einer Verbesserung der Qualität in der Patientenversorgung bei und fördert die positive Wahrnehmung der Psychiatrie nach Außen. Beides sind unverzichtbare Ziele in unserem Beruf. Deshalb muss die Arbeit dieser Kollegen von den eigenen Krankenhäusern auch in Zukunft nachhaltig unterstützt werden.

Doch wie liest sich nun dieser umfangreiche Band am besten? Hilfreich ist es zu Beginn im Inhaltsverzeichnis zu stöbern, sich eine Arbeit mit einem interessanten Titel auswählen, um dann mit dem Lesen durchzustarten. Sie werden sehen, dass es den meisten Autoren gelungen ist, ihr Anliegen kurz und gut lesbar zusammenzufassen. Das soll Sie ermutigen den Band nach der ersten Arbeit nicht beiseite zu legen, sondern weiterzulesen. Es würde uns sehr freuen, wenn möglichst viele der Befunde und Erkenntnisse sie zu eigener Forschungstätigkeit stimulierten oder vielleicht sogar in die klinische Versorgung von Patienten eingingen.

Den Autoren, die an dieser Ausgabe beteiligt sind gilt unser herzlicher Dank.

Danken möchten wir auch für das Engagement der Veranstaltungsbüros in Regensburg und Irsee und dem Team des Thieme-Verlages, die wesentlich zur Durchführung des Kongresses sowie dem Erscheinen des Sonderbandes beigetragen haben.

\section{Danksagung}

Die Herausgeber bedanken sich auch im Namen der Autoren bei den Firmen Lilly, Janssen-Cilag, Bristol-Myers Squibb und GlaxoSmithKline sehr für das großzügige Sponsoring des Kongresses. Damit wurde der Druck des vorliegenden Sonderbandes möglich. 